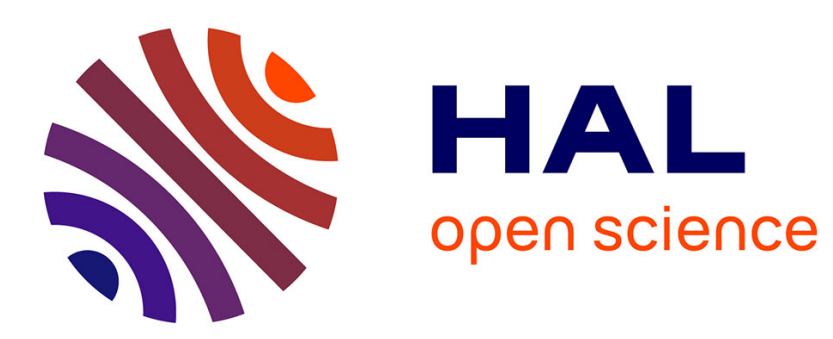

\title{
Localization and Contribution of Underwater Acoustical Sources of a Moving Surface Ship
}

Benoit Oudompheng, Barbara Nicolas, Lucille Lamotte

\section{To cite this version:}

Benoit Oudompheng, Barbara Nicolas, Lucille Lamotte. Localization and Contribution of Underwater Acoustical Sources of a Moving Surface Ship. IEEE Journal of Oceanic Engineering, 2018, 43 (2), pp.536-546. 10.1109/JOE.2017.2699260 . hal-01699595

\section{HAL Id: hal-01699595 https://hal.science/hal-01699595}

Submitted on 13 Nov 2019

HAL is a multi-disciplinary open access archive for the deposit and dissemination of scientific research documents, whether they are published or not. The documents may come from teaching and research institutions in France or abroad, or from public or private research centers.
L'archive ouverte pluridisciplinaire HAL, est destinée au dépôt et à la diffusion de documents scientifiques de niveau recherche, publiés ou non, émanant des établissements d'enseignement et de recherche français ou étrangers, des laboratoires publics ou privés. 
123

March 17, 2017

DRAFT 


\title{
Localization and contribution of underwater acoustic sources of a moving surface ship
}

\author{
Benoit Oudompheng, Barbara Nicolas Member, IEEE, and Lucille Lamotte
}

\begin{abstract}
With the advent of new standards for the regulation of acoustic radiation from ships, the measurement of the underwater acoustic radiation of surface ships has become a new concern. This paper proposes array signal-processing methods for localization and estimation of the spectral contributions of underwater acoustic-noise sources of moving surface ships. Beamforming for moving sources is used for localization of the acoustic-noise sources. Then, deconvolution of the point-spread function in the beamforming is performed to estimate the noise-source contributions in this underwater application. Among the classical methods of deconvolution in an aerial environment, the source density modeling method is chosen. However, weighting of the beamforming for moving sources is not adapted to the study of large vessels such as ships, which induces the localization of nonphysical sources. A new beamforming weighting strategy is proposed to deal with this issue. In addition, as beamforming for moving sources suffers from poor resolution at low frequencies, a passive synthetic aperture array algorithm is proposed here to successfully improve the resolution. A unique experiment was performed to validate the proposed methods, on Castillon Lake, France, with a towed surface ship and controlled noise sources. In these experiments, the localization performance of the beamforming for moving sources is improved by the synthetic aperture algorithm. Moreover, the new weighting strategy provides better estimation of the contribution of the sources from the deconvolution results.
\end{abstract}

\section{Index Terms}

Noise mapping, underwater acoustics, beamforming, pass-by noise

B. Oudompheng was with the laboratory GIPSA-Lab, 11 Rue des Mathématiques, 38402 SAINT-MARTIN D'HÈRES, France. He is now with Thales Underwater Systems SAS, Sophia-Antipolis, 06560, France. (benoit.oudompheng@sfr.fr)

B. Nicolas is now with the laboratory CREATIS, INSA Lyon, Bât. Blaise Pascal, 7 Avenue Jean Capelle, 69621 VILLEURBANNE, France (barbara.nicolas@creatis.insa-lyon.fr)

L. Lamotte is now with MicrodB, 28 Chemin du Petit Bois, CS 80210, 69134 ÉCULLY Cedex, France. (lucille.lamotte@microdb.fr) 


\section{INTRODUCTION}

In the littoral environment, the environmental impact of anthropogenic underwater noise has been assessed, and in particular that linked to the activities for the promotion of renewable energies (e.g., pile driving for offshore wind turbines [1]) and to ship traffic [2]. To regulate the noise from ship traffic, some standards have been defined to measure the underwater sound pressure level radiated by ships [3], [4]. With the adoption of the Marine Strategy Framework, a Directive of the European Commission in 2008 urged European acousticians to develop methods for monitoring [5] and reduction of shipping noise [6]. Current international standards define only single-microphone measurements of the global pass-by noise of a ship, measured in the far-field [3], [4].

Recent studies of pass-by noise in the aeronautic [7], railway [8], and automotive [9] industries have been carried out using microphone arrays. The aim of these studies was to noise-map a vehicle passing-by to:

1) obtain the localization of the different acoustic sources of the vehicle;

2) provide information about the relative contributions of these sources to the global acoustic pressure level.

The use of array processing appears to be an interesting technique for the diagnosis of surface ships, in order to reduce their underwater acoustic radiation.

The Doppler effect on moving acoustic sources means that noise mapping of a vehicle passingby is currently computed by beamforming for moving sources (beamforming-MS), which takes into account the vehicle motion[7]. Beamforming-MS aims to compensate for the Doppler effect by time resampling the measured acoustic pressures, and back-propagating the measured acoustic waves to a moving calculation grid. This grid follows the vehicle as it passes by, due to an $a$ priori known trajectory. However, the use of beamforming-MS to estimate the source level is limited in the case of multiple sources, because of the beamforming-MS point-spread function (PSF) in the localization map. To provide a good estimate of the source spectral contributions in the acoustic signature of a vehicle, deconvolution methods of the PSF are classically used[7]. Inspired by the array-processing methods developed in the aerial domain, this paper proposes an initial method for noise mapping of a ship passing-by. This noise mapping is based on existing methods and consists of beamforming-MS followed by deconvolution using the source density modeling (SDM) method[10]. The original contribution of this paper thus consists of 
two improvements to this initial method.

For short measurement distances compared to the size of a vessel, as used in the present study, the spherical spreading of the acoustic waves implies undesired amplification of the beamforming-MS results at the extremities of the calculation grid. A new weighting method is proposed here to reduce the number of nonphysical sources at the extremities of the grid. Moreover, beamforming-MS suffers from poor resolution at low frequencies, which limits the localization performance for close sources. A passive synthetic aperture array technique is proposed here to improve the localization resolution for monochromatic sources at low frequencies; e.g., the mechanical noise sources of vessels. To validate the array processings proposed here, a unique pass-by experiment with a 1:5 scale model of a surface ship was conducted using controlled noise sources, on Castillon Lake, France.

Section II presents the initial method for noise mapping of a ship passing-by using beamformingMS and the SDM deconvolution method[10]. Section III details the two improvements to the beamforming-MS: the new weighting strategy, and the passive synthetic aperture array algorithm. Section IV concerns the experimental applications and improvements to the initial method, while section $\mathrm{V}$ draws our conclusions from this study and provides future insight.

\section{The INITIAL METHOD}

This section presents the chosen method, which is initially computed to provide a first estimate of the spatial acoustic signature of a ship. It is based on existing array-processing techniques proposed in aerial acoustics. The estimation of the spatial acoustic signature is performed by first localizing the noise sources of the ship, and then estimating the energy contributions of each identified noise source.

\section{A. Localization step: The beamforming-MS algorithm}

Noise mapping of a vehicle or vessel is classically achieved by assuming that it can be modeled as a discrete distribution of point acoustic monopole sources. For the case under study, it was shown by Morse et al. [11] and Berry et al. [12] that the assumption of the modeling of the ship's hull as a distribution of monopole sources is realistic.

To perform this mapping, beamforming-MS is used [7]. This merges a de-Dopplerization step with a moving focusing step:

- The usual de-Dopplerization is achieved by time resampling the measured pressures. 
- The moving focusing is computed using steering vectors defined between the array and a moving calculation grid. The calculation grid has the same trajectory as the vessel, which is here known a priori. It is defined by the set of calculation points $X_{l}(\tau), \forall l \in[1, L]$ where the source locations are estimated, and the pressure levels estimated at these points are the amplitudes of the equivalent acoustic monopole sources.

With low Mach numbers and far-field scenarios, which are realistic in pass-by experiments of surface ships, some assumptions can be made during the short time intervals of duration $T$, which are here referred to as snapshots:

- The sources are in fixed positions at the frequencies and speeds of interest; i.e., the source displacement during a snapshot is smaller than the beamforming-MS resolution;

- The Doppler effect is negligible at the frequencies and speeds of interest; i.e., it does not exceed the frequency resolution defined for the localization results.

Under these assumptions, the beamforming-MS algorithm can be implemented in a simple way in the frequency domain. Considering an array of $M$ hydrophones located at $A_{m}, m \in[1, M]$, the measured acoustic pressures are sliced into snapshots that are indexed by $k . t^{k}$ is the time corresponding to the center of the $k^{\text {th }}$ snapshot. For a given snapshot $k$, the recorded pressure on the $m^{t h}$ receiver located at $A_{m}$ is expressed in the frequency domain as $p^{k}\left(A_{m}, f\right), \forall m \in[1, M]$. Conventional beamforming $p_{\mathrm{BF}}^{k}\left(X_{l}(k), f\right)$ is then computed using a calculation grid $X_{l}(k), \forall l \in$ $[1, L]$ that is centered at the vessel position at the time corresponding to the snapshot $k$. The beamforming-MS complex value at point $X_{l}(k)$ is moving at the same speed as the study vessel, and for the snapshot $k$ is expressed as:

$$
p_{\mathrm{BF}}^{k}\left(X_{l}(k), f\right)=\mathbf{g}^{k, H}\left(X_{l}(k), f\right) \mathbf{p}_{a}^{k}(f)
$$

where $\mathbf{p}_{a}^{k}(f)=\left[p^{k}\left(A_{1}, f\right), \ldots, p^{k}\left(A_{M}, f\right)\right]^{T}$ is the vector of the measured pressures for the snapshot $k$ and at the frequency $f,{ }^{H}$ is the conjugate transpose operator, and ${ }^{T}$ is the transpose operator. The vector $\mathrm{g}^{k}\left(X_{l}(k), f\right)=\left[g^{k}\left(A_{1}, X_{l}(k), f\right), \ldots, g^{k}\left(A_{M}, X_{l}(k), f\right)\right]^{T}$ is the concatenation of the beamforming-MS steering vectors between the sensors at points $A_{m}, m \in[1, M]$ and the calculation point $X_{l}(k)$ at the frequency $f$, and this is expressed as:

$$
\mathbf{g}^{k}\left(X_{l}(k), f\right)=w_{l}(k) \mathbf{h}^{k}\left(X_{l}(k), f\right)
$$

where $w_{l}(k)$ is the beamforming-MS weight, and the vector $\mathbf{h}^{k}\left(X_{l}(k), f\right)=\left[h^{k}\left(A_{1}, X_{l}(k), f\right)\right.$, $\left.\ldots, h^{k}\left(A_{M}, X_{l}(k), f\right)\right]^{T}$ is the concatenation of the transfer functions between the sensors at 
points $A_{m}, m \in[1, M]$ and the calculation point $X_{l}(k)$ at the frequency $f$. In this paper, the transfer function between a measuring point $A_{m}$ and a calculation point $X_{l}(k)$ corresponds to the free-space Green's function with spherical spreading:

$$
h^{k}\left(A_{m}, X_{l}(k), f\right)=\frac{\exp \left(-j 2 \pi \frac{f}{c} r_{m l}(k)\right)}{4 \pi r_{m l}(k)}
$$

where the distance $r_{m l}(k)=\left\|\overrightarrow{X_{l}(k) A_{m}}\right\|$ is between the sensor $A_{m}$ and the calculation point $X_{l}(k)$ for the snapshot $k$. The beamforming-MS weight in Equation (2) that ensures recovery of the amplitude of a monopole point source in the single source case is here expressed as for the snapshot $k$ :

$$
w_{l}^{k}(k)=\left\|\mathbf{h}^{k}\left(X_{l}(k), f\right)\right\|_{2}^{-2}=\left(\sum_{m=1}^{M}\left(4 \pi r_{m l}(k)\right)^{-2}\right)^{-1}
$$

Finally, the beamforming-MS result is calculated by averaging the energy of $p_{\mathrm{BF}}^{k}\left(X_{l}(k), f\right)$ over all of the snapshots, which is expressed as:

$$
\Gamma_{B F}\left(X_{l}, f\right)=\frac{1}{K} \sum_{k=1}^{K}\left|p_{\mathrm{BF}}^{k}\left(X_{l}(k), f\right)\right|^{2}
$$

\section{B. Contribution step: Deconvolution of the beamforming-MS results}

Considering $N$ monopole sources located at the moving points $S_{n}, n \in[1, N]$, the beamformingMS response of the excitation of these $N$ acoustic sources is theoretically:

$$
\mathbf{Z}_{\mathrm{BF}}(f)=|\mathbf{P S F}(f)|^{2} \boldsymbol{\Gamma}_{s}(f)
$$

where $|\mathbf{P S F}(f)|^{2}$ is the $L \times N$ matrix of the average PSFs over all of the $K$ snapshots, and $\Gamma_{s}(f)$ is the $N \times 1$ vector of the source autospectra. The element $(l, n)$ of the matrix $|\mathbf{P S F}(f)|^{2}$ is the value at the point $X_{l}$ of the average beamforming-MS PSF centered at the source point $S_{n}$, at frequency $f$ :

$$
|\mathbf{P S F}(f)|^{2}(l, n)=\sum_{k=1}^{K}\left|w_{l}^{k}(k) \mathbf{g}^{k, H}\left(X_{l}(k), f\right) \mathbf{g}^{k}\left(S_{n}(k), f\right)\right|^{2}
$$

where $\mathrm{g}^{k}\left(S_{n}(k), f\right)$ is the $M \times 1$ vector of the transfer functions between the sensors $A_{m}$ and the source point $S_{n}(k)$.

In the multiple-source case, an accurate estimate of the noise-source levels can be computed by deconvolution of the average PSF from the beamforming-MS results (Eq. (6)). In underwater acoustics, deconvolution algorithms have been studied to improve the estimation of the ambient 
noise directionality with a towed linear array [13], [14]. These algorithms are based on a nonnegative least-squares strategy that minimizes the quadratic error between the measured beamforming outputs and a given beamforming model. The computation concerns beamforming outputs for static sources. Deconvolution has also been performed using the Lucy-Richardson algorithm, when the PSF is position independent [15]. In these papers, results from measured data demonstrate that the deconvolution technique can be used to lower sidelobes.

The pass-by noise of aircraft in aerial environments is generally studied using beamformingMS. In this field, deconvolution algorithms are commonly used to estimate the relative contributions of each localized source to the global acoustic signature of an aircraft. For instance, in the railway industry, Bruhl developed the SDM method, which is based on a gradient descent algorithm [10], and Le Courtois adapted the deconvolution approach for the mapping of acoustic sources (DAMAS) method of Brooks et al. [16] for the case of moving sources [17]. In railway applications, because of the high speeds of trains and the short measurement distances, only the snapshot corresponding to the position of the train at the broadside of the antenna is used for the processing. Consequently, the deconvolution results obtained without time averaging are often noisy. In aeronautic applications, the measurement distances are often long, and time averaging over several snapshots is possible. In this context, Fleury et al.[7] extended the classical deconvolution methods of DAMAS, DAMAS2, CLEAN, and CLEAN-SC to their case for moving sources. The classical methods of DAMAS, CLEAN and SDM that resolve the deconvolution problem in the least-squares sense were studied on the experimental test case, as detailed in section IV. The CLEAN method was not chosen because it requires $a$ priori knowledge of the number of sources, which is unknown in practice. The DAMAS method resolves the deconvolution problem by processing sequentially the amplitude estimation for each calculation point, while the algorithm is iterative and stops when a threshold for the reconstruction error is reached. The DAMAS method appears to be sensitive to noisy environments, as it localizes many nonphysical sources. On the other hand, the SDM method iteratively resolves the deconvolution problem for the whole calculation grid. This global resolution strategy allows the number of localized nonphysical sources to be reduced. Mathematically, this corresponds to a smaller reconstruction error than DAMAS in the least-squares sense in a simulated case of two uncorrelated monopole sources in linear uniform motion. Consequently, the SDM iterative method [10] is used here for the deconvolution of the average PSF on the beamforming-MS results. 
Let $\Gamma_{x}(f)$ be the $L \times 1$ vector of the autospectra of the equivalent monopole sources located at the calculation points. The $l$-th element of the vector $\Gamma_{x}(f)$ is denoted as $\Gamma_{x}\left(X_{l}, f\right)$. The SDM method solves the inverse problem of the estimation of the noise-source autospectra by a-priori knowing a model of the average PSF (Eq. (6)) with a realistic nonnegative constraint on the unknown source autospectra[10], which is the following least mean square problem:

$$
\left\{\begin{array}{l}
\widehat{\boldsymbol{\Gamma}}_{x}(f)=\arg \min _{\boldsymbol{\Gamma}_{x}(f)}\left\|\boldsymbol{\Gamma}_{\mathrm{BF}}(f)-|\mathbf{P S F}(f)|^{2} \boldsymbol{\Gamma}_{x}(f)\right\|_{2}^{2} \\
\Gamma_{x}\left(X_{l}, f\right)>0, \forall l \in[1, L]
\end{array}\right.
$$

The algorithm of SDM is an algorithm of a projected gradient with a nonnegative constraint. This algorithm is initialized using beamforming-MS as follows:

$$
\widehat{\boldsymbol{\Gamma}}_{x}(f)^{(0)}=\boldsymbol{\Gamma}_{\mathrm{BF}}(f)
$$

At the iteration $\alpha$, the $L$ autospectra of the estimated sources at points $X_{l}, l \in[1, L]$ are:

$$
\widehat{\boldsymbol{\Gamma}}_{x}(f)^{(\alpha)}=\widehat{\boldsymbol{\Gamma}}_{x}(f)^{(\alpha-1)}
$$

$$
+2 \chi|\mathbf{P S F}(f)|^{2, H}\left(\boldsymbol{\Gamma}_{\mathrm{BF}}(f)-|\operatorname{PSF}(f)|^{2} \widehat{\boldsymbol{\Gamma}}_{x}(f)^{(\alpha-1)}\right)
$$

where $\chi$ is the speed parameter of the gradient descent that results from a balance between convergence and convergence speed. The value of $\chi$ was chosen as $5 \times 10^{-5}$ in the present study. At the end of each iteration, the nonnegative constraint on the unknown source autospectra is satisfied by turning the negative values to zero.

\section{IMPROVEMENTS TO THE INITIAL METHOD}

To study the localization of ship noise sources as carried out in section IV, the calculation grid used for the beamforming-MS method is larger than the measurement distance, because of the large size of the vessel. There is undesired amplification at the sides of the calculation grid, which degrades the localization results through the generation of nonphysical sources. Moreover, due to the limited size of the array and the measurement distance, the localization of two close sources is not possible at low frequencies.

\section{A. New weighting strategy for beamforming-MS}

For the study of large objects such as ships, near-field beamforming-MS is used and the classical weighting coefficients of beamforming-MS (Eq. (4)) amplify the estimated sources at 
the farther calculation points. For instance, in the experiment described in section IV, the ship's length is $21 \mathrm{~m}$ and the array size is $4 \mathrm{~m}$. Moreover, the measurement distance is short, to get a good signal-to-noise ratio (SNR), which implies that the minimum distance between a calculation point and a hydrophone is $10.37 \mathrm{~m}$, and the maximum distance is $19.88 \mathrm{~m}$. Beamforming-MS aims to estimate the acoustic pressure radiated at $1 \mathrm{~m}$ by an equivalent monopole source located at each calculation point. Consequently, the energy measured by the sensors and back-propagated to a distant calculation point will be amplified because of the different distances between this calculation point and the hydrophones on the array. This is particularly disturbing in the presence of strong background noise, thus degrading the localization results on the sides of the calculation grid, which can lead to incorrect interpretations.

These undesired amplifications would not exist if all of the calculation points were located at equal distances from the acoustic center of the array; i.e., on a sphere centered at the array center. Thus, for the case of spherical microphone antennas, Pereira suggested to weight the acoustic transfer functions between the measuring points and the calculation points to deal with this issue [18]. These weights are the distances between the calculation points and the array acoustic center. This technique allows the array to be insensitive to its positioning relative to the calculation points[18]. Pereira demonstrated this observation with the application of the inverse method, the equivalent source method. Investigations into the beamforming-MS method deal with this issue by modification of the steering vectors and not using any weights like those described in Equation (2). In these other studies, the steering vector between the $m^{\text {th }}$ sensor and the $l^{\text {th }}$ calculation point at the frequency $f$ is:[10], [16], [19]:

$$
g_{0}\left(A_{m}, X_{l}(k), f\right)=\frac{r_{m l}(k)}{r_{c l}(k)} \exp \left(-j 2 \pi \frac{f}{c} r_{m l}(k)\right)
$$

where $r_{c l}(k)=\left\|\overrightarrow{A_{c} X_{l}\left(t^{k}\right)}\right\|$, is the distance between the array center $A_{c}$ and the calculation point $X_{l}(k)$ for the snapshot $k$.

In this paragraph, we propose to use Pereira's strategy, which consists of weighting the transfer function associated to the calculation point $X_{l}(k)$ by the distance $r_{c l}(k)$ for the snapshot $k$. This choice allows the expression of the beamforming-MS steering vector as a function of the transfer function, to maintain a physical meaning for the steering vector. The free-field transfer function with a spherical spreading is given by Equation (3). Let us define a function $h_{\mathrm{ref}}^{k}$ that depends on the free-field transfer function as follows:

$$
h_{\mathrm{ref}}^{k}\left(A_{m}, X_{l}(k), f\right)=r_{c l}\left(t^{k}\right) h^{k}\left(A_{m}, X_{l}(k), f\right)
$$


where $A_{m}$ is the $m^{t h}$ sensor point, $X_{l}$ is the $l^{\text {th }}$ calculation point for the frequency $f$. The

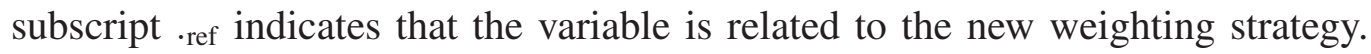

The normalization term $w_{l, \text { ref }}(k)$ of the beamforming-MS using the new transfer function is now:

$$
w_{l, \mathrm{ref}}(k)=\left\|\mathbf{h}_{\mathrm{ref}}^{k}\left(X_{l}(k), f\right)\right\|_{2}^{-2}
$$

with $\mathbf{h}_{\mathrm{ref}}\left(X_{l}(k), f\right)=\left[h_{\mathrm{ref}}\left(A_{1}, X_{l}(k), f\right), \ldots, h_{\mathrm{ref}}\left(A_{M}, X_{l}(k), f\right)\right]^{T}$.

The new beamforming-MS steering vector is expressed as:

$$
\mathbf{g}^{k}\left(X_{l}(k), f\right)=w_{l, \text { ref }}(k) \mathbf{h}^{k}\left(X_{l}(k), f\right)
$$

The theoretical response of the beamforming-MS at point $X_{l}(k)$ to a monopole source of unit amplitude located at point $X_{l}(k)$ for the snapshot $k$ is now:

$$
\mathbf{g}^{k, H}\left(X_{l}(k), f\right) \mathbf{h}\left(X_{l}(k), f\right)=\frac{1}{r_{c l}(k)}
$$

This new strategy of beamforming-MS weighting mathematically corresponds to the estimation of the source amplitude at points $X_{l}(k), l \in[1, L]$, which are multiplied by $\frac{1}{r_{c l}(k)} ;$ i.e., to estimate the acoustic pressure radiated at the array center by an equivalent monopole source located at each calculation point at time $t^{k}$.

The source contribution is then estimated using the deconvolution algorithm presented in subsection II-B, and using the PSF of Equation (7). Note that if a real source is localized at a given calculation point $X_{l}(k)$ for all of the snapshots indexed by $k$, then its amplitude will be

multiplied by $\frac{1}{K} \sum_{k=1}^{K} \frac{1}{r_{c l}^{2}(k)}$ in the final result of the beamforming-MS at the point $X_{l}$ (Eq. (5)). Its amplitude can thus be recovered if the ship's trajectory is perfectly known.

\section{B. A passive synthetic aperture array algorithm for monochromatic sources}

Many studies on passive synthetic aperture arrays have been carried out over the last two decades. These studies have mostly considered the case of towed arrays. Williams performed experiments of active synthetic aperture array processing using a ship-towed source that emitted a continuous waveform signal[20]. Stergiopoulos et al. developed an efficient extended towed array method (ETAM) based on an overlap correlator that estimated the phase correction factor from the spatial overlap of virtual hydrophones with real hydrophones[21]. This correlationbased technique appeared to be robust to trajectory mismatches, because it was independent of the exact knowledge of the ship position. Other methods have benefited from beamforming 


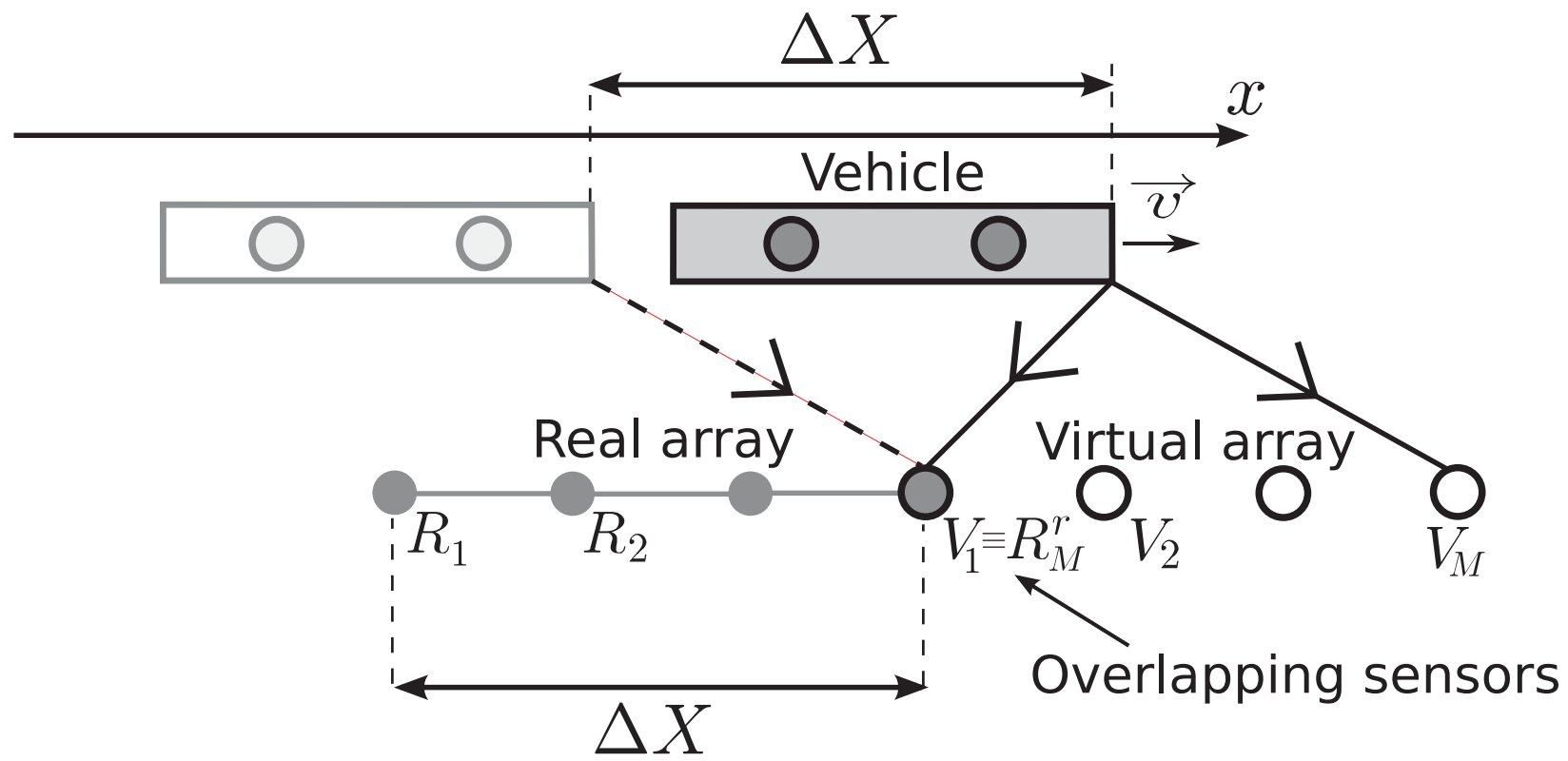

Fig. 1: Construction of the passive synthetic aperture antenna for a vessel passing-by at speed $v$.

processing to build a synthetic aperture technique. The beamforming method is a coherent array processing technique that is thus more robust in low SNR experiments than when processed directly on the sensors, which is the case for ETAM. For instance, Yen et al. proposed a fast Fourier transform synthetic aperture algorithm that phase compensated the complex beamforming outputs of subaperture arrays, instead of their virtual pressures [22]. Tong et al. extended the ETAM to the case of multiple sources with different trajectories by also using beamforming outputs of subaperture arrays [23].

In this paper, we propose to build a passive synthetic aperture array according to the ETAM, to improve the resolution of the localization method for monochromatic sources at low frequencies [24].

1) Initial passive synthetic aperture array algorithm: The initial synthetic aperture array algorithm is implemented following the following two steps[20]:

1) Definition of subaperture arrays from real measured pressures that correspond to subtrajectories;

2) Synchronization of the pressures measured by the virtual arrays with the pressures measured by the real array. 
Let us assume an initial real linear array $\left\{R_{m}\right\}_{m=1 . . M}$ and a virtual array built at a distance of $\Delta X$ from the real array position. The vehicle displacement is parallel to the array axis, i.e., the $\mathrm{X}$-axis. This distance $\Delta X$ corresponds to the source displacement during the delay $\xi$ (Fig. 1). The virtual pressures measured by the synthetic array $\left\{V_{m}\right\}_{m=1 . . M}$ are expressed as:

$$
p\left(V_{m}, t\right)=\mathcal{F}^{-1}\left[\mathcal{F}\left[p\left(R_{m}, t-\xi\right)\right](f) \exp \left(j \Phi_{m}\left(f_{s}\right)\right)\right](t)
$$

where $\mathcal{F}[$.$] is the time Fourier transform, f_{s}$ is the frequency of the monochromatic source, and $\Phi_{m}\left(f_{s}\right)$ is the phase shift that is necessary to synchronize the virtual sensor $V_{m}$ with the corresponding real sensor $R_{m}$. In the case of linear uniform source motion, the time delay is simply expressed as:

$$
\xi=\frac{\Delta X}{v}
$$

The compensating phase factor is then expressed as[20]:

$$
\Phi_{m}\left(f_{s}\right)=2 \pi f_{s} \frac{\Delta X}{v}
$$

2) Application of the extended towed array method: For the robustness of the synchronization of the synthetic arrays, Stergiopoulos et al. suggested to compute this using the intercorrelation between the real hydrophones and the overlapping virtual hydrophones [21]. This synchronization strategy aims to improve the estimation of the compensating phase shift $\Phi_{m}\left(f_{s}\right)$. For a virtual hydrophone $V_{m}$ built from a real hydrophone $R_{m}$ and located at the position of a real overlapping hydrophone $R_{n}$, the phase correction factor is estimated by:

$$
\Phi_{m}\left(f_{s}\right)=2 \pi f_{s} \arg \max _{u} \int_{t} p\left(R_{m}, t-\xi\right) p\left(R_{n}, t-u\right) d t
$$

where $\arg \max _{u}$ is the time $u$ corresponding to the maximum.

To be robust to any changes in the propagation conditions during the passing-by of the source (e.g., nonuniform source speed, nonstationarity of the properties of the propagation medium), the construction of the passive synthetic aperture array is computed with a short time strategy. Using the snapshots (indexed by $k$ ) defined for the beamforming-MS, the short time virtual pressure $p^{k}\left(V_{m}, t\right)$ for the snapshot $k$ measured by a synthetic sensor $V_{m}$ is expressed as:

$$
p^{k}\left(V_{m}, t\right)=\mathcal{F}^{-1}\left[\mathcal{F}\left[p^{k}\left(R_{m}, t-\xi\right)\right](f) \exp \left(j \Phi_{m}^{k}\left(f_{s}\right)\right)\right](t)
$$

where $p^{k}\left(R_{m}, t\right)$ is the short time pressure measured by the real sensor $R_{m}$ and the short time correction phase factor:

$$
\Phi_{m}^{k}\left(f_{s}\right)=2 \pi f_{s} \arg \max _{u} \int_{t} p^{k}\left(R_{m}, t-\xi\right) p^{k}\left(R_{n}, t-u\right) d t
$$




\section{EXPERIMENTAL RESULTS}

The initial method and its improvements are now applied and validated on experimental data. First, the experimental set-up is presented. Then, array processing results are commented on in terms of localization results and contribution results.

\section{A. Experimental set-up}

An underwater pass-by experiment was conducted in the acoustic test facilities of DGA Naval Systems, on Castillon Lake, France, which is a mountain lake in the Alps that has an average depth of $100 \mathrm{~m}$ and a maximum width of $600 \mathrm{~m}$. The experiment consisted of towing a 21-mlong scale model of a surface ship at a constant speed of $v=2 m \cdot s^{-1}$ above a horizontal linear nine-hydrophone array.

The ship's trajectory is parallel to the array, along the x-direction. The z-axis represents the depth axis, and the coordinate system has its origin at the array center. The ship's hull has two shakers, $S_{1}$ and $S_{2}$, that generate two point acoustic sources outside the hull, as shown on Figure 2. When the ship is at the closest point of approach (CPA), the shakers are located at the points with the x-coordinates of: $x=-5.9 \mathrm{~m}$ for $S_{1}$, and $x=2.3 \mathrm{~m}$ for $S_{2}$. The hydrophone array is parallel to the $\mathrm{x}$-axis and to the ship's trajectory. The nine-element array is $4 \mathrm{~m}$ long, with $0.5-\mathrm{m}$ element spacing. The array depth is $10 \mathrm{~m}$, and the array is located at $2.50 \mathrm{~m}$ from the CPA in the y-direction (Fig. 2). The acquisition time considered for the array processing is longer than the duration of the ship's passing above the array.

The ship's trajectory is calculated using a tachymeter system positioned on the idler pulley. The tachymeter system provides a trajectory accuracy of $0.785 \mathrm{~cm}$. To correct for any possible trajectory errors, a reference shaker that emits a sinus at $5,000 \mathrm{~Hz}$ is used to localize the CPA. The CPA time is estimated by the time when the signal emitted by this reference shaker has a frequency of $5,000 \mathrm{~Hz}$, as received at the center of the array. When the ship's trajectory is not perfectly known, the amplitude of a point source will be spread over several grid points. It will thus be necessary to define large contribution zones in order to take into account this uncertainty, or to improve the trajectory estimation using [25].

This experimental set-up is a realistic configuration with good SNR, and it is used for experimental validation of the proposed method.

Note that the following analysis of the noise-mapping results is only qualitative because the amplitudes of the acoustic sources are not known. Indeed, the signals emitted by the shakers are 


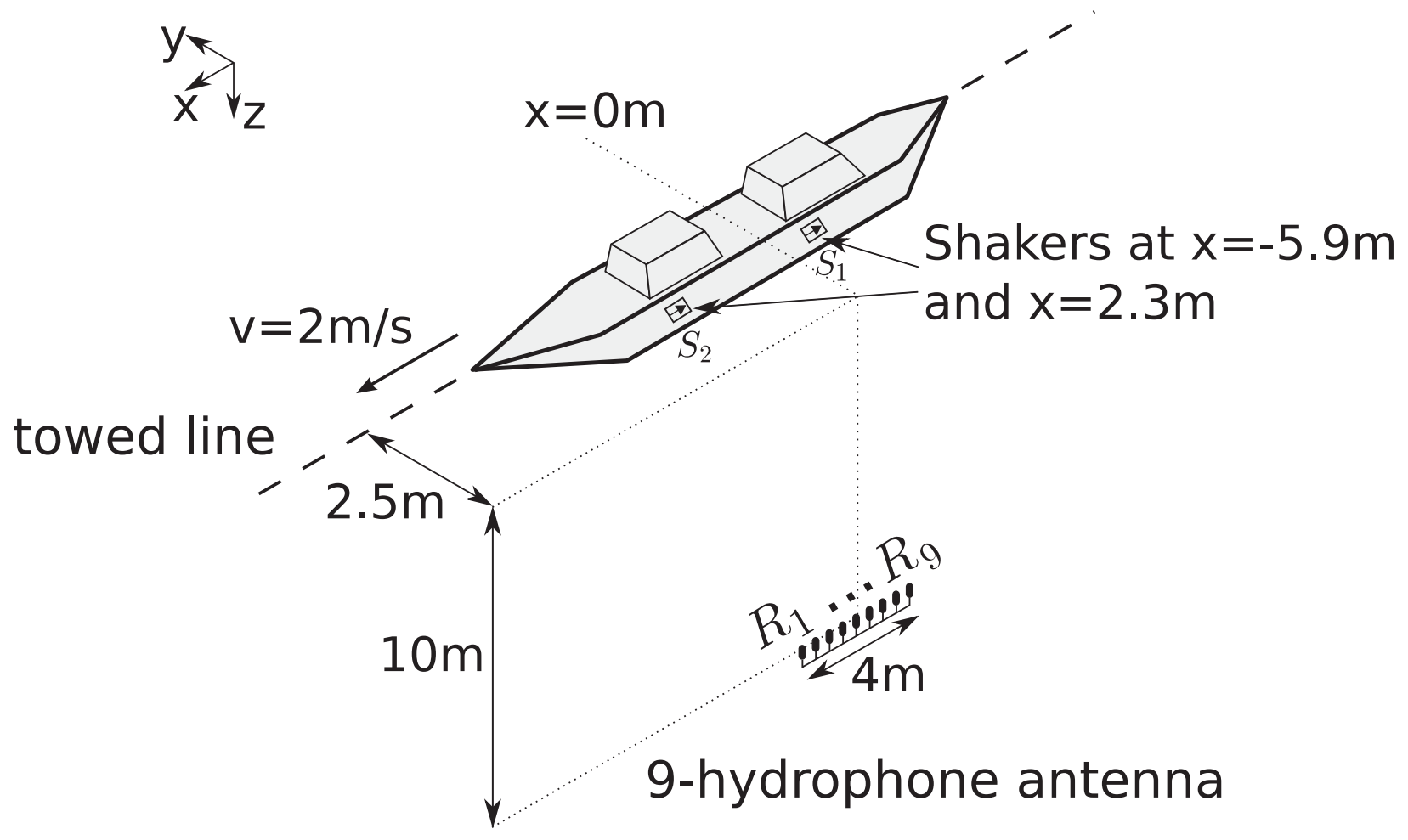

Fig. 2: Experimental set-up for the study of two moving acoustic sources on Castillon Lake.

controlled, although the vibroacoustic response of the ship's hull is not known. Consequently, the accuracy of the estimation of the relative energetic contributions of the sources cannot be verified. Different emitted signals will be used in the following: sines and wideband noise. The SNR is the mean SNR while the ship passes by.

\section{B. Application of the new strategy of weighting}

The shaker at $x=-5.9 \mathrm{~m}$ emits three sines at the frequencies of $1,200 \mathrm{~Hz}, 1,400 \mathrm{~Hz}$, and $1,800 \mathrm{~Hz}$, and it represents a mechanical source, such as an engine source. The shaker at $x=2.3$ $\mathrm{m}$ emits wideband noise that represents a hydroacoustic source, such as a propeller source. On a hydrophone of the array, the SNR is $50 \mathrm{~dB}$ for the 3 sine source, and $25 \mathrm{~dB}$ for the wideband source. The new weighting strategy is evaluated using this realistic scenario in terms of source localization and source contribution. The recording duration is $14.15 \mathrm{~s}$, which corresponds to 281 snapshots of duration $T=100 \mathrm{~ms}$, with $50 \%$ overlap, at a distance of $28.30 \mathrm{~m}$, and at a speed of $2 \mathrm{~m} \cdot \mathrm{s}^{-1}$. 
1) Source localization performances: Figure 3 shows the localization result computed by beamforming-MS using the initial weighting (Fig. 3a), and after deconvolution by SDM of this initial beamforming-MS (Fig. 3b). These localization maps represent the acoustic pressure levels in decibels as a function of the estimated source position on the horizontal axis and of the frequency on the vertical axis. The beamforming-MS and the deconvolution by SDM are computed for each frequency in the band of interest $[500 \mathrm{~Hz} ; 2,000 \mathrm{~Hz}]$. The true source positions are indicated by the vertical dashed lines and by the small arrows in the pictures of the ship models at the bottom of Figure 3. With the initial weighting, the two sources are roughly localized in the good source areas by the beamforming-MS with bad resolution at low frequencies, and by the SDM method with better resolution. However, after deconvolution by SDM, several nonphysical sources at undesired localizations are spread over all of the localization map, and particularly at the ends of the calculation grid. These nonphysical sources correspond here to deconvolution residues of the beamforming-MS sidelobes due to mismatches between the theoretical PSF (Eq. 7) and the real PSF.

Figure 4 shows the localization results computed by beamforming-MS using the new weighting (Fig. 4a) and after deconvolution by SDM of this beamforming-MS result (Fig. 4b), with the same parameters as previously used. The two sources are localized at their real positions by both methods, which was not the case with the initial beamforming-MS. These results highlight the interest in the new weighting strategy for the provision of more accurate localization of the sources. In addition, the nonphysical sources that were previously localized at the extremities of the calculation grid have now been removed (Fig. 4). This result validates the interest in the new weighting strategy.

However, the wideband source $S_{2}$ at $x=2.3 \mathrm{~m}$ is not continuously localized over all of the frequencies, and especially at the frequencies of the source $S_{1}$, which has a $15-d B$-higher amplitude at the hydrophone. This limitation is imposed by the localization dynamic of the SDM method, which is almost the same as the one of the beamforming-MS.

2) Source contribution performances: To estimate the relative contributions of the sources in the acoustic signature, the contribution zones must be defined. Three zones are defined, based on the deconvolution results:

- 'Zone 1', which corresponds to shaker $S_{1}$ at $x=-5.9 \mathrm{~m}:[-8 m ;-4 m]$

- 'Zone 2', which corresponds to shaker $S_{2}$ at $x=2.3 \mathrm{~m}$ : $[0 \mathrm{~m} ; 4 \mathrm{~m}]$

- Zone 'residue', which corresponds to nonphysical sources (i.e., outside 'Zone 1' and 'Zone 


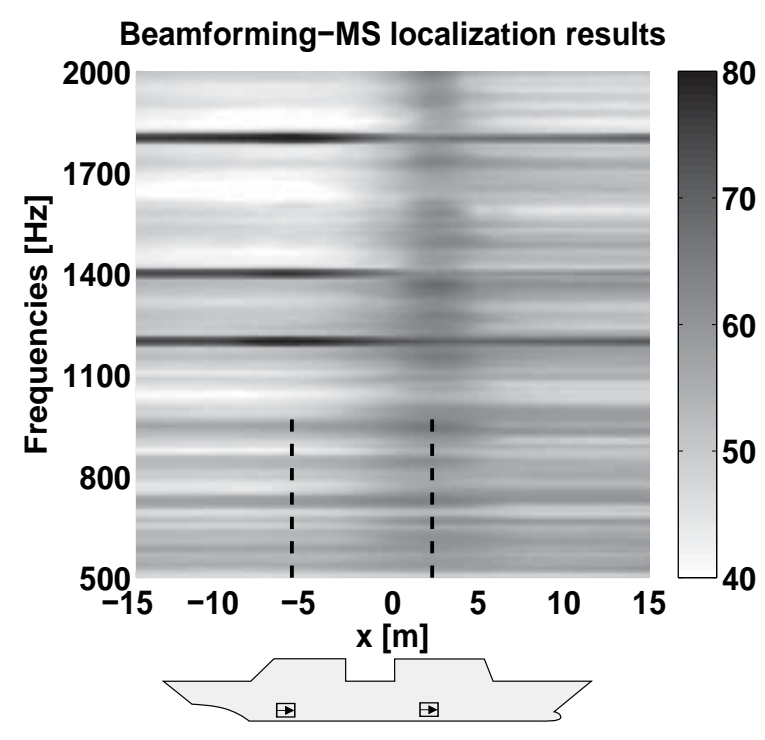

(a) Beamforming-MS

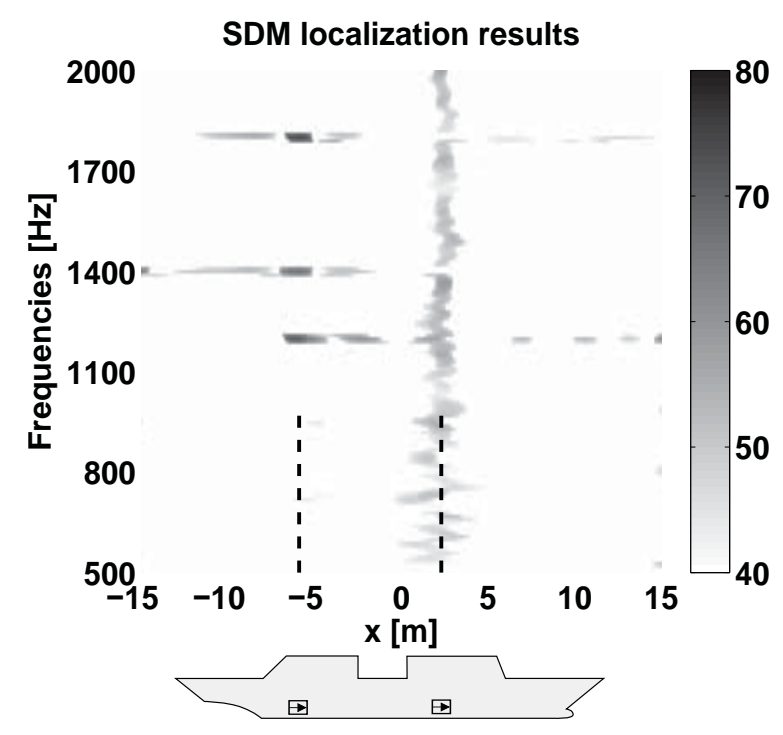

(b) SDM

Fig. 3: Localization maps representing the estimated pressure levels in acoustic decibels, as a function of the estimated source position and of the frequency. (a) Result of the beamforming-MS with the initial weighting. (b) Result from the beamforming-MS after deconvolution by SDM. The source positions are indicated by the vertical dashed lines and by the arrows in the pictures of the ship models at the bottom of the panels.

$$
\text { 2'): }[-15 m ;-8 m] \cup[-4 m ;+0 m] \cup[+4 m ; 15 m]
$$

The contribution spectrum of a zone is computed by spatial integration of the spectra estimated after SDM deconvolution for all of the calculation points included in this studied zone. Figure 5 represents the estimated contribution spectrum of source $S_{1}$ as the continuous line, of source $S_{2}$ as the dashed line, and of nonphysical sources as the dotted line. The results shown in Figure 5a do not allow correct estimation of the source contributions. Indeed, the level of the residue contribution is only $10 \mathrm{~dB}$ lower than the contribution of source $S_{2}$. Figure $5 \mathrm{~b}$ shows that the new beamforming-MS weighting improves the estimation of the spectral contribution of source $S_{1}$. Indeed, the acoustic energy of the estimated source $S_{1}$, which is represented as the continuous line on Figure 5, is concentrated only on the frequencies of 1,200 Hz, 1,400 Hz, and $1,800 \mathrm{~Hz}$ in the results with the new weighting (i.e., Fig. 5b). Moreover, the energy contribution of the nonphysical sources referred to as 'residue' is dramatically reduced, by more than $10 \mathrm{~dB}$, 


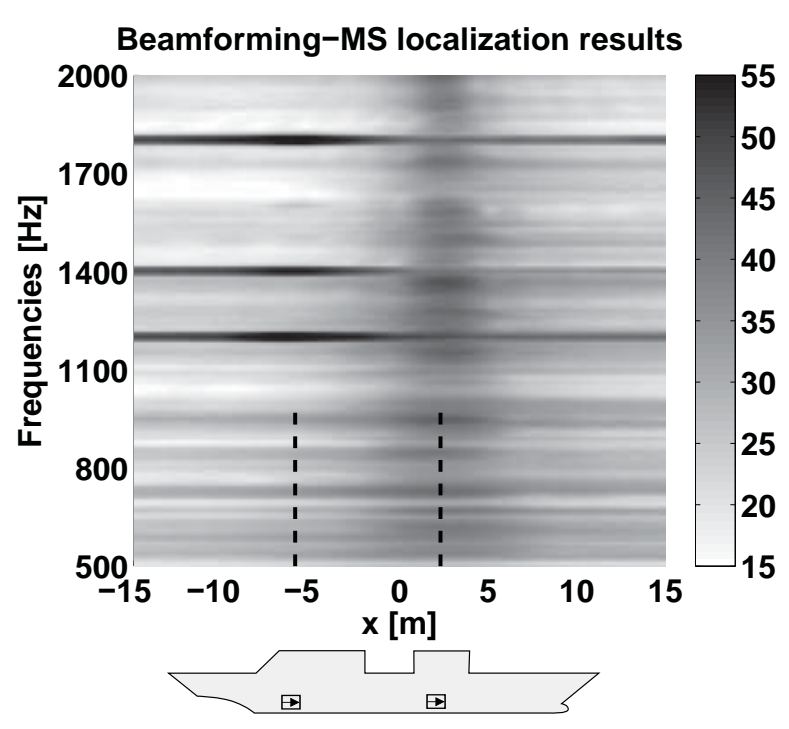

(a) Beamforming-MS

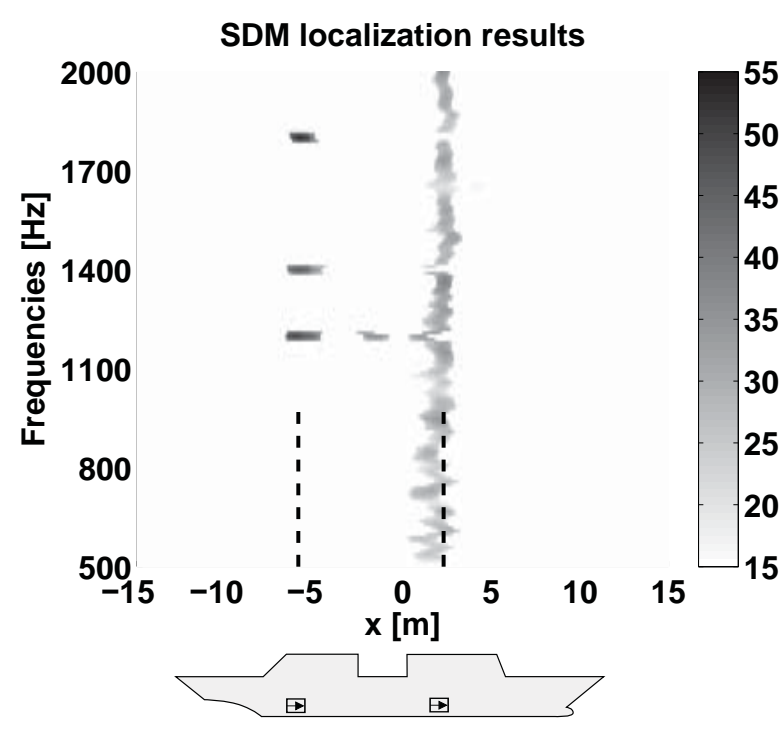

(b) SDM

Fig. 4: Localization maps representing the estimated pressure levels in acoustic decibels, as a function of the estimated source position and of the frequency. (a) Result of the beamformingMS with the new weighting. (b) Result from the beamforming-MS after deconvolution by SDM. The source positions are indicated by the vertical dashed lines and by the arrows in the pictures of the ship models at the bottom of the panels.

in the result computed with the new weighting (i.e., Fig. 5b) compared to that computed with the initial weighting (i.e., Fig. 5a). This observation means that the spectral contributions of the two sources are better estimated using the new weighting.

In conclusion, these experimental results show that the initial method with the new weighting strategy provides accurate localization of the sources of a ship passing-by. In addition, the deconvolution results are improved by the new weighting, thus providing good estimation of the relative energy contributions of these sources. The main improvement from the new weighting strategy is the drastic reduction in the nonphysical sources.

\section{Application of synthetic aperture pre-processing}

For this second validation test case, the two shakers emitted two sinusoidal signals at a frequency $f_{s}=700 \mathrm{~Hz}$ (Fig. 2). The SNR is $60 \mathrm{~dB}$. This configuration can occur when two 


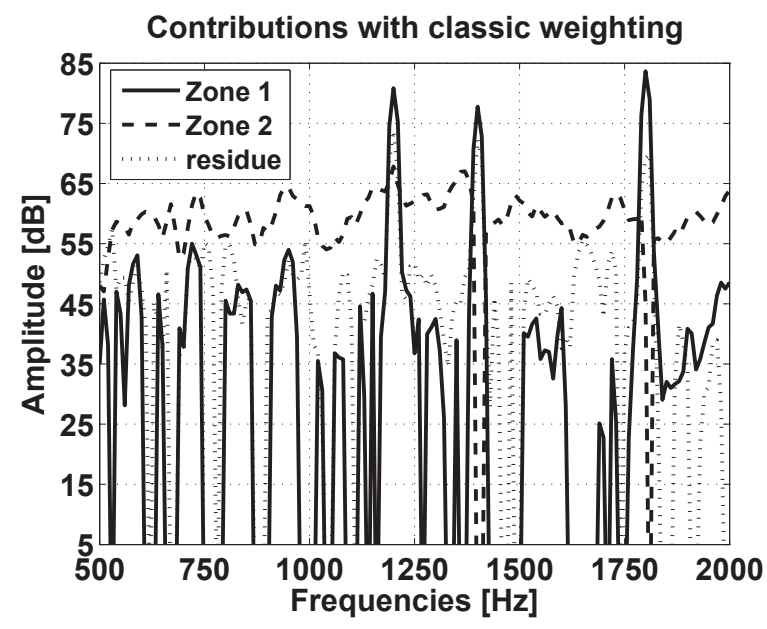

(a) Initial weighting

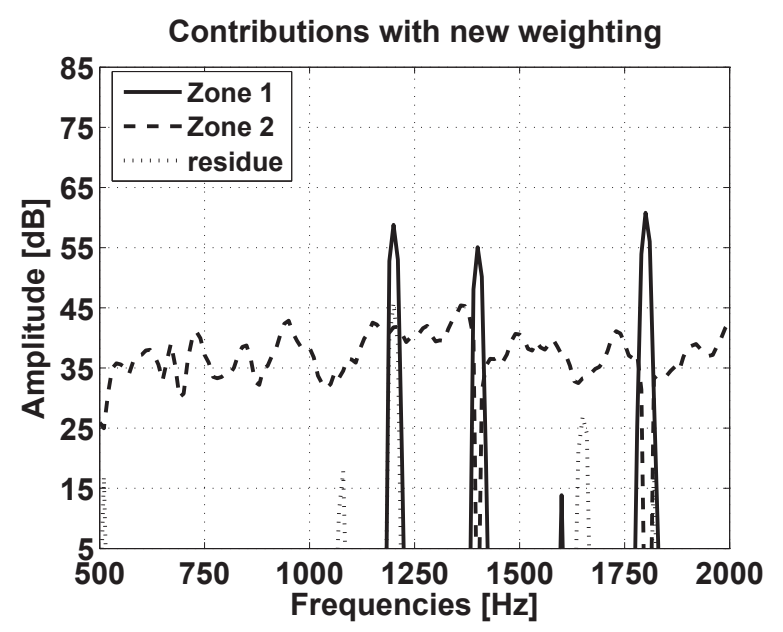

(b) New weighting

Fig. 5: Estimated contribution spectra of source $S_{1}$ as the continuous line, source $S_{2}$ as the dashed line, and nonphysical sources as the dotted line, with the initial weighting (a) and the new weighting (b).

mechanical sources are close, such as for two internal machines. The recording duration is $7 \mathrm{~s}$, which corresponds to 139 snapshots of duration $T=100 \mathrm{~ms}$ with $50 \%$ overlap.

1) Construction of the synthetic array: The real array of nine hydrophones, $\left\{R_{m}\right\}_{1 . .9}$ is synthetically enlarged by twice its length. The choice of the synthetic aperture length is important, and it depends mainly on the source and antenna directivities. Empirically, it has been shown that a maximum angle of view of $45^{\circ}$ between the ship and the array provides satisfactory results. The total synthetic array, $\left\{\left\{V_{m}\right\}_{1 . .4},\left\{R_{m}\right\}_{1 . .9},\left\{V_{m}\right\}_{7 . .10}\right\}$, has 17 hydrophones with a virtual length of $8 \mathrm{~m}$. Figure 6 illustrates the geometry of the synthetic array. The synthetic array is enlarged by defining two sub-arrays of five virtual hydrophones with an overlap of one hydrophone. The pairs of overlapping sensors are $\left(V_{5} ; R_{1}^{r}\right)$ and $\left(V_{6} ; R_{9}^{r}\right)$.

These pairs of overlapping hydrophones are useful for robust synchronization (Eq. (21)), and also for experimental assessment of the quality of the construction of the synthetic antenna. For this purpose, two indicators in the literature[26] are calculated here for a pair of overlapping sensors $\left(R_{n}^{r} ; V_{m}\right)$, where - refers to the average over time, and superscript.$^{r}$ denotes the overlap:

- Indicator $T_{1}$ : A correlation coefficient between the overlapped hydrophone time pressures that evaluates the quality of the phase compensation $\left(T_{1}=1\right.$ means that the signals are in 


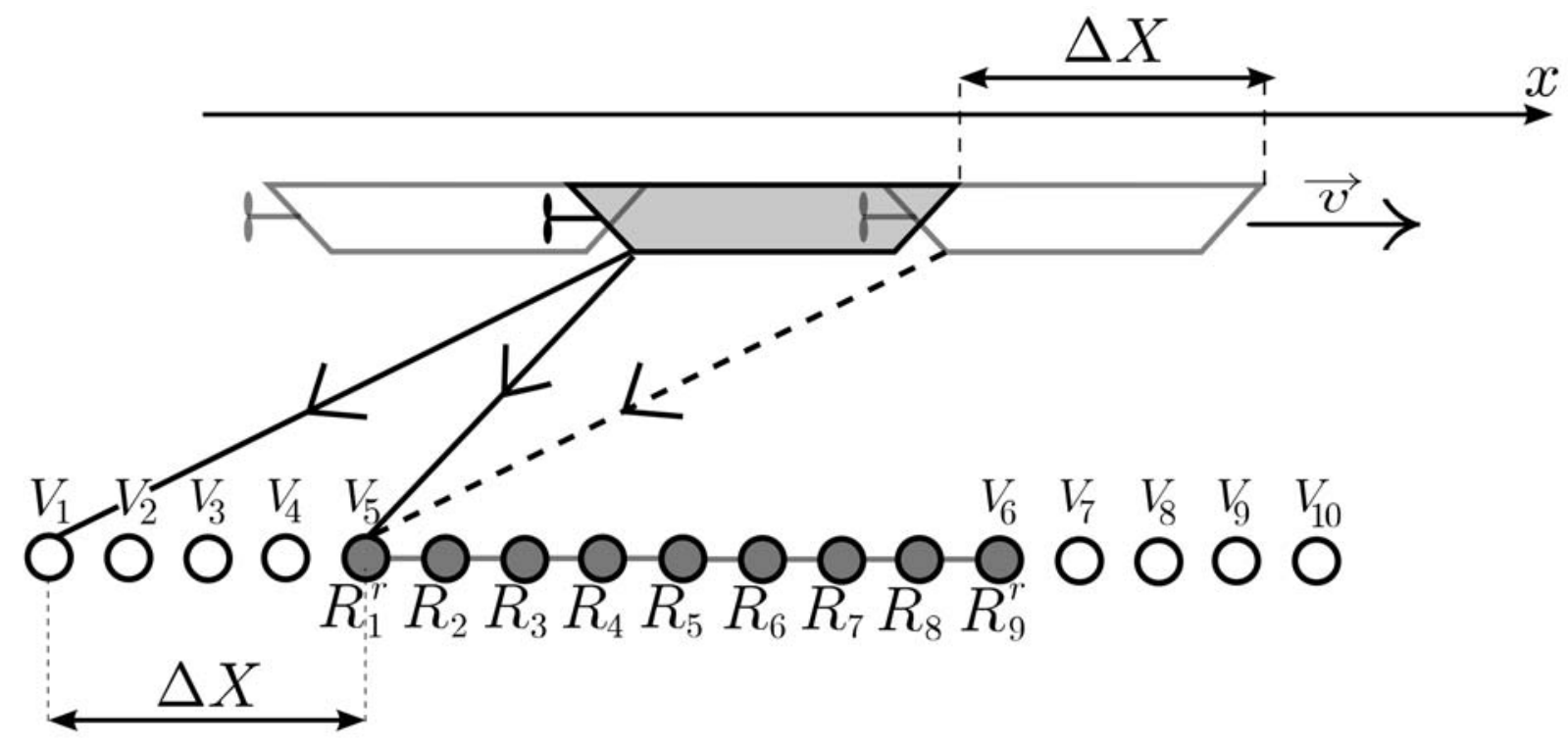

Fig. 6: Construction of the synthetic array of 17 hydrophones $\left\{\left\{V_{m}\right\}_{1 . .4},\left\{R_{m}\right\}_{1 . .9},\left\{V_{m}\right\}_{7.10}\right\}$ from the real antenna of nine hydrophones $\left\{R_{m}\right\}_{1 . .9}$.

phase):

$$
T_{1}=\frac{\overline{p\left(V_{m}, t\right) \cdot p\left(R_{n}^{r}, t\right)}}{\sqrt{\overline{p\left(V_{m}, t\right)^{2} p\left(R_{n}^{r}, t\right)^{2}}}}
$$

- Indicator $T_{2}$ : The relative error between the root mean square of the overlapped hydrophone time pressures ( $T_{2}$ should tend to zero):

$$
T_{2}=\frac{\left|\sqrt{\overline{p\left(R_{n}^{r}, t\right)^{2}}}-\sqrt{\overline{p\left(V_{m}, t\right)^{2}}}\right|}{\sqrt{\overline{p\left(R_{n}^{r}, t\right)^{2}}}}
$$

Figure 7 shows the values of both of the indicators for the pair of overlapping hydrophones $\left(V_{5} ; R_{1}^{r}\right)$ (Fig. 7a) and $\left(V_{6} ; R_{9}^{r}\right)$ (Fig. 7b) as a function of time $t^{k}, \forall k \in[1, K]$, which are the times corresponding to snapshots. Note that the indicators are computed for the samples of the snapshots defined in subsection II-A. All of the indicators $T_{1}$ are close to 1 , and all of the indicators $T_{2}$ are close to 0 , so it can be concluded that the synthetic aperture array is built correctly.

2) Source-localization performances: The previous experimental indicators show the good quality of the synchronization of the virtual hydrophones with the real hydrophones. The sourcelocalization results are now computed using beamforming-MS and the deconvolution SDM 


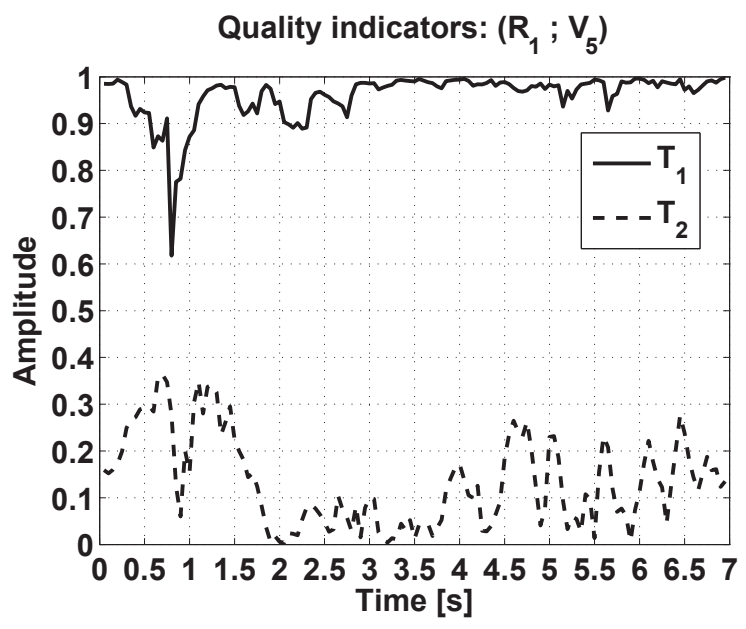

(a) $\left(V_{5} ; R_{1}^{r}\right)$

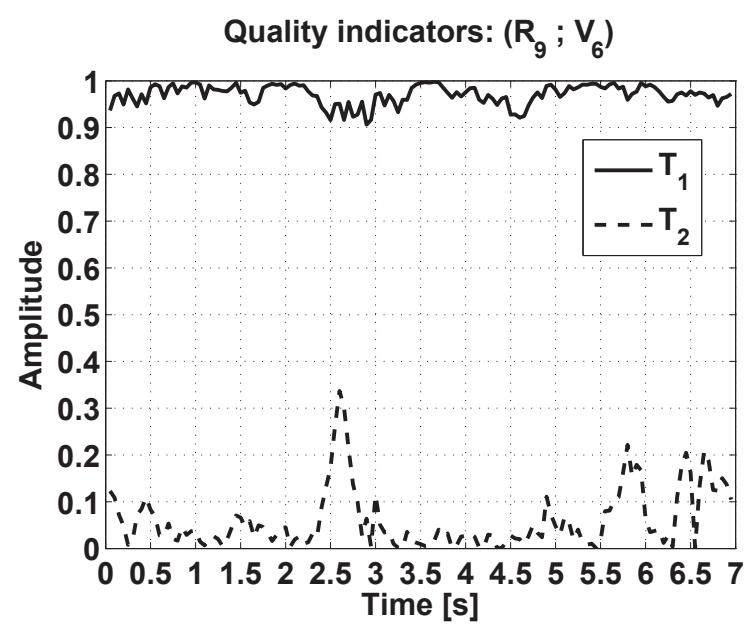

(b) $\left(V_{6} ; R_{9}^{r}\right)$

Fig. 7: Values of the quality indicators $T_{1}$, as the continuous line, and $T_{2}$, as the dashed line, for the pairs of overlapping sensors $\left(V_{5} ; R_{1}^{r}\right)$ (a) and $\left(V_{6} ; R_{9}^{r}\right)$ (b).

method, to evaluate the improvements to the localization at low frequencies. Figure 8 shows the localization results at $700 \mathrm{~Hz}$ with the real nine-hydrophone array as the continuous line, and with the synthetic 17-hydrophone array as the dashed line, as computed by the beamformingMS (Fig. 8a) and after deconvolution by the SDM method (Fig. 8b).

Figure 8a shows that the beamforming-MS improves the localization results using the 17hydrophone synthetic array. Quantitatively, Table I presents the data for the width of the main lobe at $-3 d B$ of the maximum (i.e., the resolution), the side-lobe level, and the localization errors for each source and each array. The values of the resolution confirm that the synthetic array that has a length twice that of the real array (which implies two-fold narrower resolution) is in agreement with the theory. In addition, both sources are localized with small errors (of less than $1 \mathrm{~m}$ ) using the 17-hydrophone synthetic array, as compared to the beamforming-MS using the nine-hydrophone real array, which only localizes one source. These localization errors are due to phase errors that probably result from a small error in the construction of the virtual array. For instance, the indicator $T_{1}$ of the couple $\left(V_{5} ; R_{1}^{r}\right)$ is low for the first snapshots, which means that the phase compensation is not accurate. Another probable explanation is that the sidelobes of each source might slightly bias the localization of the other one. The localization result with the 17-hydrophone synthetic array informs the operator that there may be two sources. After 


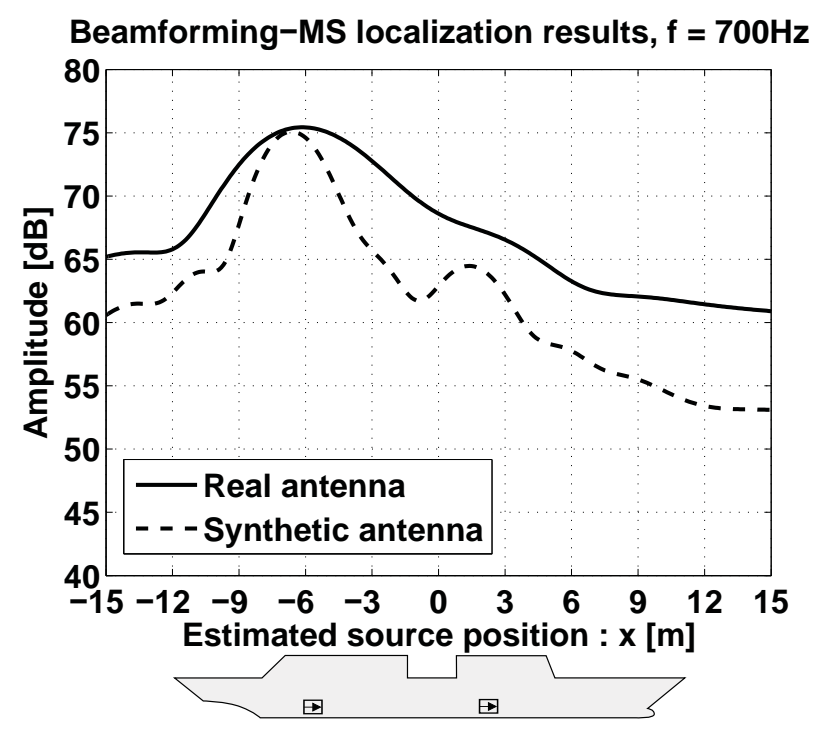

(a) Beamforming-MS

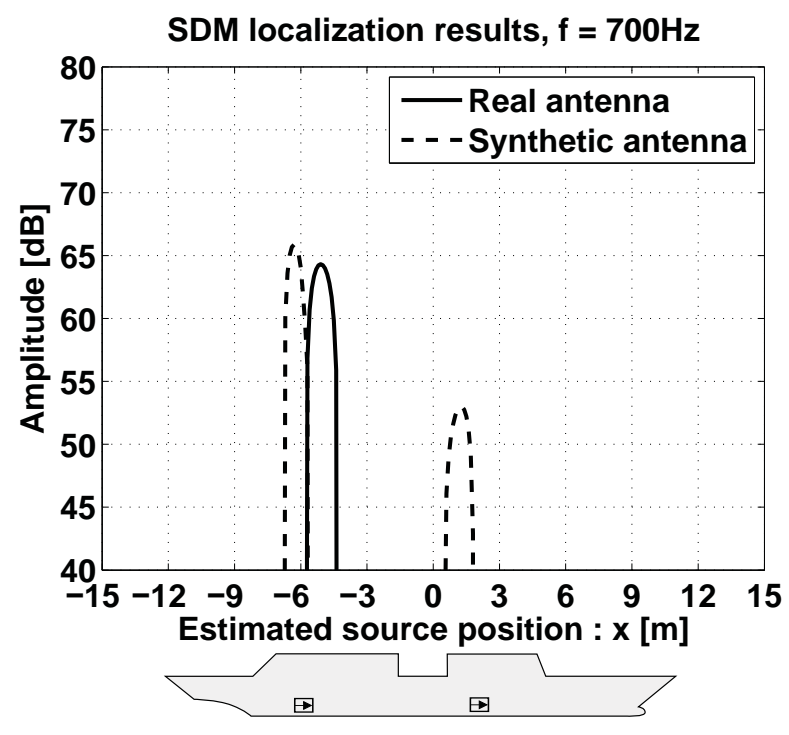

(b) SDM

Fig. 8: Source-localization results with the real array as the continuous line and the synthetic antenna as the dashed line, for the beamforming-MS (a) and from the beamforming-MS after deconvolution by SDM (b).

deconvolution by the SDM method (Fig. 8b), both of the sources are localized with certainty with the 17-hydrophone synthetic aperture antenna. No nonphysical source is localized by the SDM method, which allows accurate contribution estimation using the SDM results.

In conclusion, this experiment with the two sinusoidal sources at low frequency demonstrates that the synthetic aperture array can be used to improve the performance of beamforming-MS, and especially of its resolution. The synthetic aperture array allows here the localization of both sources with certainty after deconvolution, which was not the case with the real antenna. This is of great interest for naval applications, as ships have numerous low-frequency tonal sources.

\section{CONCLUSiOnS AND FURTHER STUdies}

This paper proposes a noise-mapping process to localize noise sources and to estimate their contributions for the case of a moving surface ship above a horizontal linear array. The initial array-processing method is the beamforming-MS method computed in the Fourier domain, for source localization, and for deconvolution of its result by the SDM method, to estimate the spectral contributions of the sources. The choice of the initial method was motivated by an 


\begin{tabular}{|c|c|c|c|}
\hline $\begin{array}{c}\text { Type of } \\
\text { antenna }\end{array}$ & $\begin{array}{c}\text { Primary lobe } \\
\text { beamwidth }\end{array}$ & $\begin{array}{c}\text { Side lobe } \\
\text { level }\end{array}$ & $\begin{array}{c}\text { Estimation } \\
\text { errors along } \\
\text { the x-axis }\end{array}$ \\
\hline $\begin{array}{c}\text { Real } \\
\text { (9 hydro.) }\end{array}$ & $6.2 \mathrm{~m}^{*}$ & $*$ & $*$ \\
\hline $\begin{array}{c}\text { Synthetic } \\
\text { (17 hydro.) }\end{array}$ & $3.2 \mathrm{~m}\left(\hat{S}_{1}\right)$ & $11.07 \mathrm{~dB}\left(\hat{S}_{1}\right)$ & $-0.7 \mathrm{~m}\left(\hat{S}_{1}\right)$ \\
& $3.8 \mathrm{~m}\left(\hat{S}_{2}\right)$ & $6.28 \mathrm{~dB}\left(\hat{S}_{2}\right)$ & $-0.9 \mathrm{~m}\left(\hat{S}_{2}\right)$ \\
\hline
\end{tabular}

TABLE I: Performances of the localization by beamforming-MS with the computation of a synthetic aperture antenna. * As the two sources are not localized with the real array, the performances concerning the estimated source $S_{1}$ are not relevant.

analysis of the literature in other areas of application. In the case of the pass-by experiment, the study of a large vessel implies that all of the points are at very different distances from the antenna. Consequently, there are undesired amplifications of the estimated pressure levels at the extremities of the calculation grid in the localization results, due to spherical spreading. To deal with this issue, a new weighting strategy for the beamforming-MS is proposed. Moreover, a surface ship has many low-frequency sources that correspond to the machinery noise, and this initial method fails to separate two close sources at low frequencies even after deconvolution. The use of the passive synthetic aperture array method is thus proposed for monochromatic sources, to improve the resolution of beamforming-MS at low frequencies.

To experimentally validate the improvements to the initial method, an underwater noisemapping experiment of a moving ship was conducted on Castillon Lake, France, with a horizontal linear array of nine hydrophones. The application of the new weighting strategy on a configuration of two sources shows a dramatic reduction in the number of nonphysical sources. The localization results and the contribution results are thus more accurate, which helps in their physical interpretation. Moreover, an experiment with two sinusoidal sources at low frequency 
is considered. The use of the synthetic aperture array method allows the localization of both of the sources with the synthetic antenna, which is not possible with the real antenna. The synthetic aperture array processing improves the localization performances of the beamformingMS, especially in terms of its resolution and side-lobe levels.

Future studies will be designed to generalize the initial method to all types of movement, and particularly for higher speeds, for which the Doppler effect has to be taken into account[27], [7], [28]. The deconvolution method should be modified accordingly. Recent advances in signal processing have been achieved in terms of the development of efficient blind deconvolution methods using sparsity constraints[29]. These methods appear to be adapted to this application, because of the uncertain knowledge of the PSF that depends on the knowledge of the environment, and because of the sparse distribution of the sources inside the ship. In addition, the passive synthetic aperture array should be extended to noise-map random sources, because this processing is currently implemented for monochromatic sources. Finally, the methods and their improvements should be applied to a real ship at sea.

\section{ACKNOWLEDGMENTS}

This work was financially supported by the Ministère du Redressement Productif (Direction Générale de la Compétitivité, de l'Industrie et des Services) and by the DGA-MRIS, grant RAPID ARMADA n¹22906030. The authors would like to thank DGA Naval Systems for their help to perform the experiments.

\section{REFERENCES}

[1] H. Bailey, B. Senior, D. Simmons, J. Rusin, G. Picken, and P. M. Thompson, "Assessing underwater noise levels during pile-driving at an offshore windfarm and its potential effects on marine mammals," Marine Pollution Bulletin, vol. 60, no. 6, pp. 888-897, 2010.

[2] I. H. McQuinn, V. Lesage, D. Carrier, G. Larrivée, Y. Samson, S. Chartrand, R. Michaud, and J. Theriault, "A threatened beluga (delphinapterus leucas) population in the traffic lane: Vessel-generated noise characteristics of the saguenay-st. lawrence marine park, canada," The Journal of the Acoustical Society of America, vol. 130, no. 6, pp. 3661-3673, 2011. [Online]. Available: http://scitation.aip.org/content/asa/journal/jasa/130/6/10.1121/1.3658449

[3] ASA, S12.64-2009 Quantities and procedures for description and measurement of underwater sound from ships - Part 1: General requirements, Acoustical Society of America Std., 2009.

[4] ISO, ISO/PAS 17208-1:2012 - Quantities and procedures for description and measurement of underwater sound from ships - Part 1: General requirements for measurements in deep water, ISO Std., 2012.

[5] T. Folegot, C. Gervaise, Y. Stéphan, D. Clorennec, and B. Kinda, "Now casting anthropogenic ocean noise in high pressure areas," in Acoustics 2012, 2012. 
[6] C. Audoly, C. Rousset, and T. Leissing, "Aquo project-modelling of ships as noise source for use in an underwater noise footprint assessment tool," in INTER-NOISE and NOISE-CON Congress and Conference Proceedings, vol. 249 , no. 7. Institute of Noise Control Engineering, 2014, pp. 862-871.

[7] V. Fleury and J. Bulté, "Extension of deconvolution algorithms for the mapping of moving acoustic sources," The Journal of the Acoustical Society of America, vol. 129, p. 1417, 2011.

[8] B. Barsikow, W. King, and E. Pfizenmaier, "Wheel/rail noise generated by a high-speed train investigated with a line array of microphones," Journal of Sound and Vibration, vol. 118, no. 1, pp. 99-122, 1987.

[9] H. Kook, G. Moebs, P. Davies, and J. Bolton, "An efficient procedure for visualizing the sound field radiated by vehicles during standardized passby tests," Journal of Sound and Vibration, vol. 233, no. 1, pp. 137-156, 2000.

[10] S. Bruhl and A. Roder, "Acoustic noise source modelling based on microphone array measurements," Journal of sound and vibration, vol. 231, no. 3, pp. 611-617, 2000.

[11] P. M. Morse and K. U. Ingard, Theoretical acoustics. Princeton University Press, 1968.

[12] A. Berry, J.-L. Guyader, and J. Nicolas, "A general formulation for the sound radiation from rectangular, baffled plates with arbitrary boundary conditions," The Journal of the Acoustical Society of America, vol. 88, no. 6, pp. 2792-2802, 1990.

[13] R. A. Wagstaff, "Iterative technique for ambient-noise horizontal-directionality estimation from towed line-array data," The Journal of the Acoustical Society of America, vol. 63, no. 3, pp. 863-869, 1978.

[14] J. S. Rogers and J. L. Krolik, "Time-varying spatial spectrum estimation with a maneuverable towed array," The Journal of the Acoustical Society of America, vol. 128, no. 6, pp. 3543-3553, 2010. [Online]. Available: http://dx.doi.org/10.1121/1.3505121

[15] T. C. Yang, "On conventional beamforming and deconvolution,” in OCEANS 2016 - Shanghai, April 2016, pp. 1-5.

[16] T. Brooks and W. Humphreys, "A deconvolution approach for the mapping of acoustic sources (damas) determined from phased microphone arrays," Journal of Sound and Vibration, vol. 294, no. 4, pp. 856-879, 2006.

[17] F. Le Courtois, F. Poisson, J.-H. Thomas, and J.-C. Pascal, "Sound source characterization on a high speed train from microphone array measurements," in INTER-NOISE and NOISE-CON Congress and Conference Proceedings, vol. 2011, no. 3. Institute of Noise Control Engineering, 2011, pp. 3757-3762.

[18] A. Pereira, "Acoustic imaging in enclosed spaces," Ph.D. dissertation, Laboratoire de Vibro-acoustique, INSA Lyon, 2013.

[19] S. Guérin, C. Weckmüller, and U. Michel, "Beamforming and deconvolution for aerodynamic sound sources in motion," in 1st Berlin Beamforming Conference, 2006, pp. 21-22.

[20] R. Williams, "Creating an acoustic synthetic aperture in the ocean," The Journal of the Acoustical Society of America, vol. 60, no. 1, pp. 60-73, 1976.

[21] S. Stergiopoulos and E. Sullivan, "Extended towed array processing by an overlap correlator," The Journal of the Acoustical Society of America, vol. 86, no. 1, pp. 158-171, 1989.

[22] N.-C. Yen and W. Carey, "Application of synthetic-aperture processing to towed-array data," The Journal of the Acoustical Society of America, vol. 86, no. 2, pp. 754-765, 1989.

[23] P. Tong, S. Lim, S. Chia, and J.-M. Passerieux, "Extended towed array measurement: Beam-domain phase estimation and coherent summation," in OCEANS 2006-Asia Pacific. IEEE, 2006, pp. 1-6.

[24] B. Oudompheng, B. Nicolas, and L. Lamotte, "Passive synthetic aperture array to improve noise mapping of a moving ship," in MTS/IEEE OCEANS Conference, 2015.

[25] — - "Acoustic trajectory correction to improve mapping of moving sources," in 171th meeting Acoustical Society of America, Salt Lake City, USA, 2016. 
[26] S. Paillasseur, J.-H. Thomas, and J.-C. Pascal, "Regularization for improving the deconvolution in real-time near-field acoustic holography," The Journal of the Acoustical Society of America, vol. 129, no. 6, pp. 3777-3787, 2011.

[27] H. Camargo, "A frequency domain beamforming method to locate sound sources," Ph.D. dissertation, Virginia Tech, 2010.

[28] J. Ballesteros, E. Sarradj, M. Fernandez, T. Geyer, and M. Ballesteros, "Noise source identification with beamforming in the pass-by of a car," Applied Acoustics, vol. 93, pp. 106-119, 2015.

[29] A. Repetti, M. Pham, L. Duval, E. Chouzenoux, and J.-C. Pesquet, "Euclid in a taxicab: Sparse blind deconvolution with smoothed regularization," IEEE Signal Processing Letters, vol. 22, no. 5, pp. 539-543, 2015. 